\title{
Overview of Radar Data Compression
}

\author{
Valliappa Lakshmanan \\ Cooperative Institute of Mesoscale Meteorological Studies \\ University of Oklahoma \& National Severe Storms Laboratory
}

\begin{abstract}
Radar data is routinely transmitted in real-time from the coterminous United States (CONUS) radar sites and placed on the Internet for incorporation into nowcasting, hydrology and modeling applications in near-realtime. Radar data are also archived for on-demand retrieval by the National Climate Data Center (NCDC).

Data compression is used operationally to reduce bandwidth and storage requirements. There are several data compression techniques used operationally on radar data. Custom compression techniques have been devised for radar data that outperform generic techniques but the radar operations groups ultimately use off-the-shelf solutions.

The underlying ideas behind compressibity are useful beyond just reducing the amount of data for transmission and archival. The compressibility of radar data has been found useful for devising quality control algorithms, especially for the detection and removal of test patterns.
\end{abstract}

Keywords: weather radar, compression

\section{OPERATIONAL RADAR DATA}

The current generation of radars used operationally in the United States by the National Weather Service are the Weather Surveillance Radar 1998 Doppler (WSR-88D). There are 142 operational WSR-88D radars in the coterminous United States (CONUS), with an additional 11 radars in Hawaii and Alaska. This paper will focus on the CONUS radar network. These are S-band radars, chosen for their low attenuation properties, and are capable of a surveillance (radar reflectivity-range only) of $920 \mathrm{~km}$ and a Doppler (both reflectivity and velocity data) of up to $230 \mathrm{~km}$. The WSR-88D radars scan the atmosphere at a given elevation angle, then tilt upwards and scan again. The elevations range from about 0.45 degrees to about 19.5 degrees. The radars operate in one of several predefined volume coverage patterns (VCPs) which specify the elevation angles and pulse repetition frequencies used. For example, VCP 21 (See Figure $1 ;^{1}$ ) is a common pattern chosen during severe weather situations. Currently, the resolution of radar data is $1 \mathrm{~km}$ in range and approximately 1 degree in azimuth.

\subsection{Size}

The raw radar data ("Level I") consists of multiple data values collected at a range gate. The Level I data are used for signal processing at the radar site, but are not distributed operationally outside the radar site. Instead, the processed signals are distributed as "moment" data, called Level II. The Level II data comprises a value of radar reflectivity, velocity toward/away from the radar and spectrum width (a measure of the spread of velocity values) for every range gate. Additional radar products, such as mesocyclone detections and vertically integrated products and together termed Level III, are generated from the Level II data at the radar site.

Send correspondence to lakshman@ ou.edu 


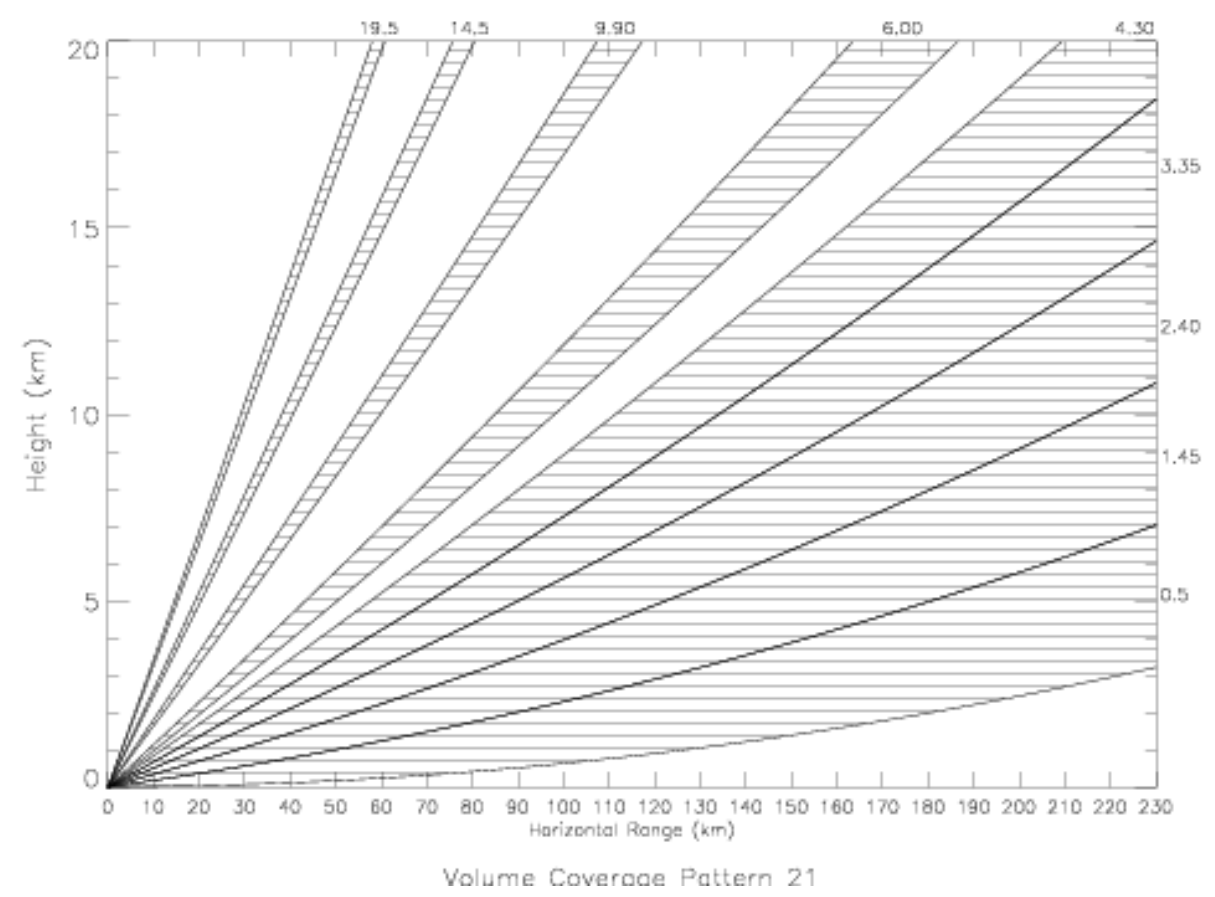

Figure 1. Volume coverage pattern (VCP) 21 of the WSR-88D. The VCP defines a regular scanning strategy and is commonly applied to mechanically scanning radars in operational settings.

The size of the radar data depends on which level of radar data is of interest. In this paper, we will focus on Level II, since that is the best data that is routinely transmitted. In many cases, superior versions of the Level III products can be generated off-site and in real-time from Level II data from multiple radars and other sensors. ${ }^{2}$

The size of the radar data also depends on the volume coverage pattern. Clear air VCPs have fewer elevation scans (there is no vertical structure to sense) and are collected slower (the radar is more sensitive and is then able to sense fine lines and boundaries). Thus, for example, a complete volume scan collected in VCP 31 contains 5 elevations, takes about 990 seconds to collect and about 7.1 MB to store. A day's worth of clear air data from a single radar would take about $0.6 \mathrm{~GB}$ to store. To transmit the data uncompressed would require a bandwidth of 7.3 kilobytes/s.

VCPs used in weather situations have more elevation scans (to sense a vertical structure) and are collected more rapidly (to track storm evolution better). Thus, for example, a complete volume scan collected in VCP 12 contains 14 elevations, takes about 460 seconds to collect and about $15 \mathrm{MB}$ to store. A day's worth of storm data from a single radar would take about 2.8 GB to store. To transmit this data uncompressed would require a bandwidth of 33.4 kilobytes/s.

\section{DATA TRANSMISSION AND STORAGE}

The data collected at the radars is transmitted over a dedicated line to the National Weather Service Forecast Office (WFO: see Figure 2). The weather forecasters at the WFO control the radar by changing its VCP, requesting a sector scan or specifying the list of Level-III products to be routinely produced. The products can then be visualized at the forecaster workstation.

The Level-II data are tapped at the WFO using the Radar Interface and Data Distribution System (RIDDS; ${ }^{4}$ ) and transmitted over the NWS network (NWSNet: see Figure 2) to one of four regional servers located at National Weather Service Regional Headquarters. ${ }^{5}$ These regional servers are linked to the Abilene (Internet 2) network on which are also linked a set of "top-tier" providers (Oklahoma, Purdue and Maryland). Using Local Data Manager (LDM) software developed by Unidata, users of the data may request data from one of these top-tier retransmitters, or from a retransmitter further down the hierarchy that happens to be receiving the desired data. 


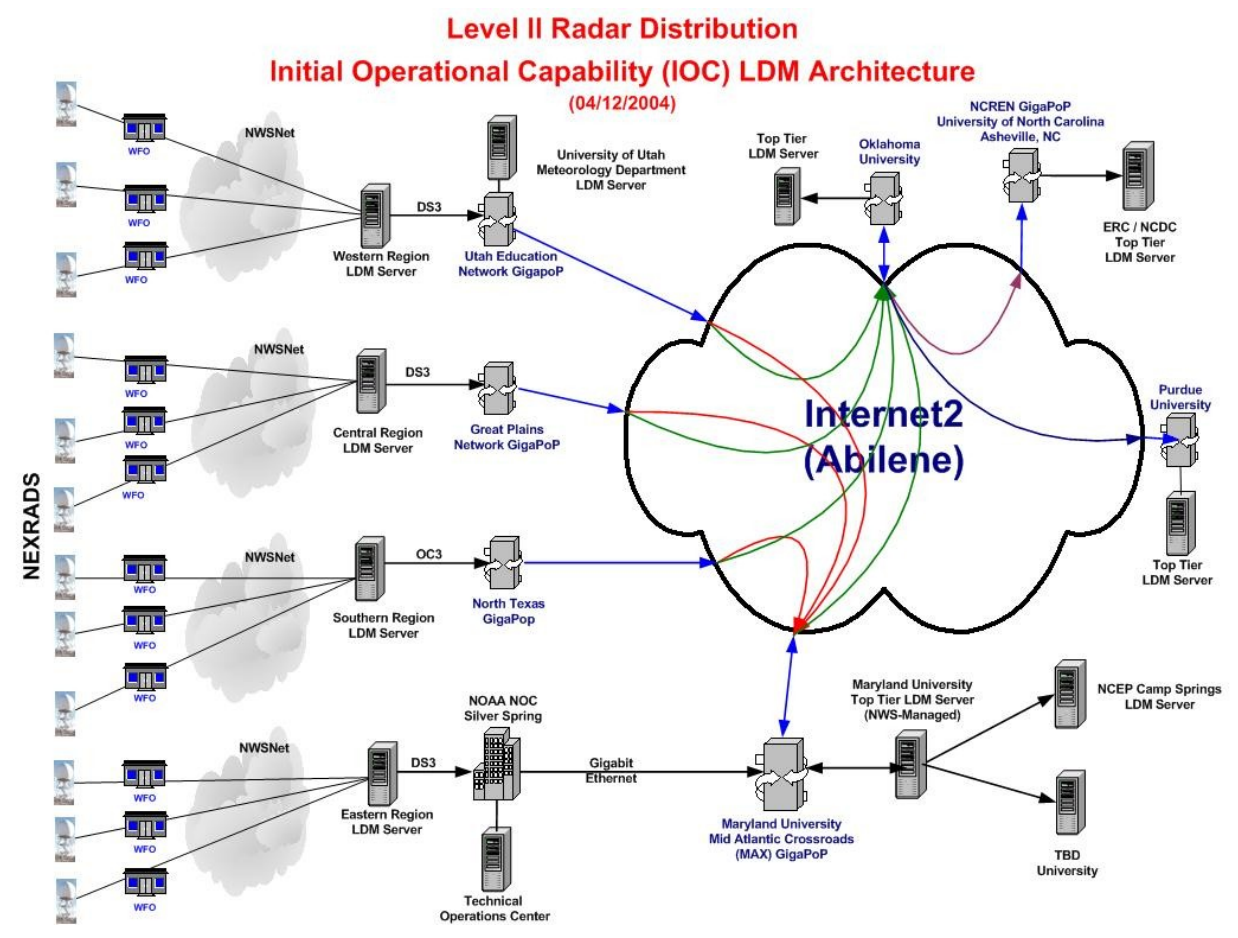

Figure 2. Real-time transmission and dissemination of radar data. ${ }^{3}$

\subsection{Bandwidth}

The actual storage and network bandwidth depends on the number of radars used, the VCPs they are in and the weather being experienced. Of course, one would not transmit uncompressed data. Instead, the data are compressed prior to first transmission and uncompressed at the point of use. With compression, the average bandwidth required to transmit all the coterminous radar over a one-year period was 735.6 kilobytes/s, with a peak bandwidth of 1770.6 kilobytes/s seen. ${ }^{3}$ This translates to an storage requirement of about $60.6 \mathrm{~GB}$ for a day's worth of radar data from the entire country. The Southern region of the United States tends to experience the most weather; the bandwidth requirement to transmit or receive all the Southern region data was about 484 kilobytes/s on average. This may be contrasted with about 74 kilobytes/s for the Eastern and Western regions. ${ }^{3}$

\subsection{Radar Data Compression}

The radar data are not transmitted in complete volume scans, since that would introduce a systematic delay in the transmission of data. Instead, they are packaged into packets of radials (typically 50 radials at a time). These packets are then compressed and placed into a LDM queue.

Custom compression strategies have been devised for radar data. For example ${ }^{6}$ examined a technique that consisted of doing a linear prediction of valid data values followed by Huffman coding of the errors in the linear prediction model. The technique takes advantage of the Markov nature of radar data with its extremely high spatial correlation. However, the improvement gained by using this custom compression algorithm was only on the order of $10 \%$ (See Figure 3, and compare the LP+BSH column to the LP-RLM column). In practice, this slight increase in compression performance is not worth the increased costs in implementing and maintaining the compression code at all end-users' sites. Therefore, the compression used in operations is the open-source and freely available bzip 2 program ${ }^{7}$ which does block sorting and achieves a performance about $10 \%$ worse than a custom compression algorithm for radar data. 


\begin{tabular}{||l|r|r|r|r|r|r|r||}
\hline \hline Elevation & Raw & RLE & RLE + LZ & LP & LP+BSH & LP-RLM & LP-RLM+BSH \\
\hline \hline 0.5 & 169280 & 146020 & 61167 & 66497 & 47998 & 43456 & 43660 \\
\hline 2.4 & 131008 & 58214 & 27263 & 35879 & 21900 & 19078 & 19460 \\
\hline 3.3 & 98624 & 40904 & 19198 & 26121 & 15629 & 13272 & 13679 \\
\hline 4.3 & 79488 & 30862 & 14346 & 20186 & 11824 & 9847 & 10281 \\
\hline 5.2 & 79272 & 26306 & 12170 & 18610 & 10004 & 8373 & 8794 \\
\hline 6.2 & 64592 & 23014 & 10552 & 15455 & 8668 & 6919 & 7375 \\
\hline 7.5 & 50279 & 20184 & 9723 & 13247 & 8201 & 6958 & 7247 \\
\hline 8.7 & 46609 & 18062 & 8728 & 11962 & 7332 & 6227 & 6456 \\
\hline 10.0 & 40370 & 16294 & 7931 & 10509 & 6612 & 5693 & 5893 \\
\hline 12.0 & 36600 & 14292 & 6910 & 9252 & 5767 & 5016 & 5160 \\
\hline 14.0 & 32850 & 12810 & 6221 & 8127 & 5070 & 4474 & 4597 \\
\hline 16.7 & 29120 & 11394 & 5582 & 7068 & 4481 & 3971 & 4029 \\
\hline 19.5 & 25340 & 10202 & 4933 & 6241 & 4012 & 3618 & 3628 \\
\hline
\end{tabular}

Figure 3. Comparison of different compression techniques on one volume scan of radar data, from. ${ }^{6}$

\subsubsection{Preprocessing}

What does help is preprocessing the radar data prior to transmission. Two particularly useful preprocessing steps are carried out.

Firstly, at the signal processor, data where the received power is less than some signal-to-noise threshold are flagged and assigned a senitel value in all the Level-II fields. Secondly, radar data corresponding to a height above mean sea level of about $20 \mathrm{~km}$ is not useful. Thus, the last few gates of the radials of higher elevation scans are zeroed out prior to invoking bzip2. While both of these technically constitute lossy compression, this is a loss that is acceptible in the context of radar data of the atmosphere.

Even though such preprocessing is carried out at the radar site, the actual compression program used on the transmitted data is bzip2. Therefore, end users do not need to implement their own software to uncompress the data.

\subsection{Archival}

One of the users of the data is the National Climatic Data Center which receives the data in realtime, uncompresses the bzipped data and builds files of complete volume scans. These volume scan files containing raw Level-II radar data are then compressed using the Unix "compress" program, an implementation of the algorithm described by. ${ }^{8}$ If the realtime transmission links fail, the forecast office can copy the files off their hard drives to tape, send it to NCDC and still get the data archived. Thus, compressed radar data volume scan files for the entire CONUS are available for public download at NCDC a couple of days later.

\section{COMPRESSIBILITY}

As the previous section described, devising custom radar compression algorithms that outperform easily available implementations like gzip ${ }^{9}$ and bzip $2^{7}$ is possible but not practically useful. However, compression research enables other goals, besides the straight-forward application to reducing networking and storage requirements.

Not all radar data corresponds to true weather. Because radar data is collected frequently and processed almost exclusively by automated algorithms, it is important to devise automated ways of identifying and rejecting bad data. An example of an instrument problem is shown in Figure 4a. To reject such instrument artifacts, it is enough to realize that such an elevation scan has almost no information content (even though many of the pixels in the scan have valid values) while good radar data as shown in Figure $4 \mathrm{~b}$ does have information (even though many of the pixels in the scan exhibit no power return). Computing Shannon's entropy measure ${ }^{10}$ on the elevation scans and rejecting elevation scans where the entropy measure falls by a large amount from the previous value is enough to reject elevation scans that exhibit instrument artifacts. ${ }^{11}$ 

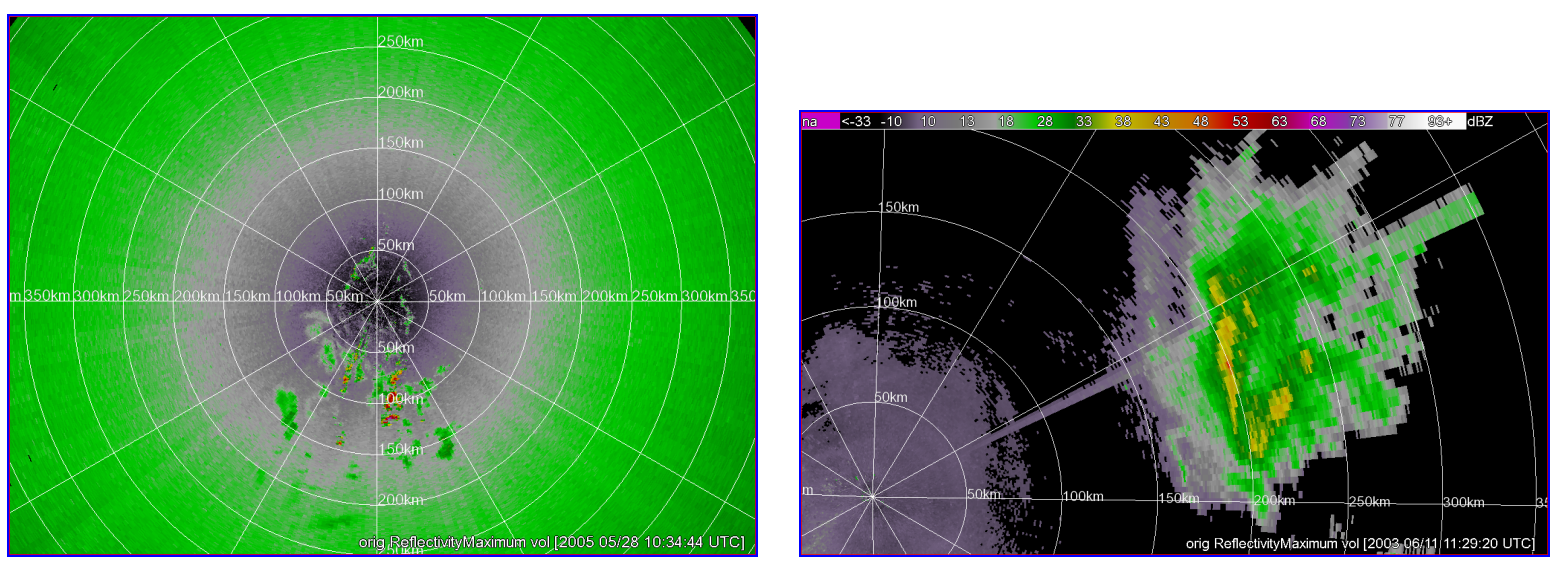

Figure 4. (a) Instrument artifact (b) Good data

\section{SUMMARY}

Radar data are compressed in radial packets after preprocessing to remove unnecessary values. The compression techniques used are commonly used programs such as UNIX compress and bzip2, so as to enable easy decoding of transmitted or retreived data. The preprocessing removed insignificant values and values at meteorologically uninteresting locations. Research efforts are better directed at finding innovative uses for compressibility ideas than ekeing out incremental improvements in the storage and bandwidth.

\section{REFERENCES}

1. T. Smith, "Visualization of WSR-88D data in 3d using application visualization software," in 14th Conf. on Weather Forecasting, Amer. Met. Society, (Dallas, TX), 1995.

2. V. Lakshmanan, T. Smith, G. J. Stumpf, and K. Hondl, "The warning decision support system - integrated information," Weather and Forecasting 22(3), pp. 596-612, 2007.

3. I. R. A. D. Services, "Level ii radar distribution," Apr 2004. https://www.irads.net/.

4. M. Jain and D. Rhue, "WSR-88D's live data stream now available in real-time," Tech. Rep. 11, National Severe Storms Laboratory, Summer 1995.

5. K. Droegemeier, K. Kelleher, T. Crum, J. Levit, S. D. Greco, L. Miller, C. Sinclair, M. Benner, D. Fulker, and H. Edmon, "Project CRAFT: A test bed for demonstrating the real time acquisition and archival of WSR-88D Level II data," in Preprints, 18th Int'l Conf. on Inter. Inf. Proc. Sys. (IIPS) for Meteor., Ocean., and Hydr., pp. 136-139, Amer. Meteor. Soc., (Orlando, Florida), 2002.

6. V. Lakshmanan, "Lossless coding and compression of radar reflectivity data," in 30th International Conference on Radar Meteorology, pp. 50-52, American Meteorological Society, (Munich), July 2001.

7. M. Burrows and D. Wheeler, "A block-sorting lossless data compression algorithm," Tech. Rep. 124, Digital Equipment Corporation, Palo Alto, California, May 1994. Available via http from gatekeeper.dec.com/pub/DEC/SRC/researchreports/abstracts/src-rr-124.html.

8. J. Ziv and A. Lempel, "A universal algorithm for sequential data compression," IEEE Trans. on Information Theory IT23, pp. 337-343, May 1977.

9. J.-L. Gailly, “Gzip.” http://www.gzip.org, 2000.

10. C. Shannon, "A mathematical theory of communication," Bell System Tech. J. 27, pp. 379-423, July 1948.

11. V. Lakshmanan, A. Fritz, T. Smith, K. Hondl, and G. J. Stumpf, "An automated technique to quality control radar reflectivity data," J. Applied Meteorology 46, pp. 288-305, Mar 2007. 